\section{Nucleus Gracilis}

John E. Mendoza

Department of Psychiatry and Neuroscience, Tulane Medical School and SE Louisiana Veterans Healthcare System, New Orleans, LA, USA

\section{Synonyms}

Gracile nucleus

\section{Definition}

Nucleus located in the lower, dorsal portion of the medulla. These paired nuclei lie on either side of the midline, just medial to the cuneate nuclei. Each nucleus represents the rostral termination of the fasciculus gracilis, the dorsal column spinal pathways that carry information regarding proprioception, fine tactual discrimination (stereognosis) and vibratory sensations from the lower extremities and lower trunk. In turn, each nucleus gives rise to internal arcuate fibers which cross the midline of the medulla and, along with similar crossing fibers from the nucleus cuneatus, form the medial lemniscus which terminates in the thalamus. As expected given its connections, a lesion in the lower and dorsal portion of the medulla affecting this nucleus would be expected to result in disturbances of proprioception, stereognosis and vibratory sense in the ipsilateral lower extremity.

\section{Cross-References}

- Fasciculus Gracilis

Medial Lemniscus 